\title{
Consequences of the Loss of Traditional Knowledge: The risk of injurious and toxic plants growing in kindergartens
}

\author{
Vanesa Pérez Cuadra, Viviana Cambi, María de \\ los Ángeles Rueda, and Melina Calfuán
}

\section{Education}

\begin{abstract}
The plant kingdom is a producer of poisons from a variety of toxic species. Nevertheless prevention of plant poisonings in Argentina is disregarded. As children are more affected, an evaluation of the dangerous plants present in kindergartens, and about the knowledge of teachers in charge about them, has been conducted. Floristic inventories and semi-structured interviews with teachers were carried out at 85 institutions of Bahía Blanca City. A total of 303 species were identified, from which 208 are considered to be harmless, 66 moderately and 29 highly harmful. Of the moderately harmful, $54 \%$ produce phytodematitis, and among the highly dangerous those with alkaloids and cyanogenic compounds predominate. The number of dangerous plants species present in each institution varies from none to 45 . Kindergartens have no landscaping plan and the majority of teachers ignore the existence of toxic plants. Appropriate actions integrating education, prevention and valuation of the natural environment are needed.
\end{abstract}

\section{Introduction}

Ancient civilizations knew which plants were safe for human consumption and which were dangerous (Álvarez Arias 2000, Neuwinger 2004). This knowledge, transmitted orally from generation to generation, has almost been lost. As early as biblical times the fruits of some trees were associated with the concept of life, whereas others with death (Wagstaff 2008), underlining the close relationship between the history of toxic plants and traditional medicine (Al-Qura'n 2005). For a long time, nature was the only source of drugs, but, since the industrial revolution, people began to prefer synthetic products for pharmacological treatments. These changes took place in association with new habits of life, especially in highly industrialized western societies, where natural drugs are consid- ered an option for people with poor education or low economic status or simply as a religious superstition (Rates 2001).

Man has always been attracted to plants whether for their beauty or economic use (source of food, fibers, dyes, etc.) but the idea that they might be harmful for health is actually uncommon (Turner \& Szcawinski 1991, Wagstaff 2008). However, poisonings by plants in humans represent a significant percentage of toxicological consultations (Córdoba et al. 2003, Nelson et al. 2007).

Although most plants do not have any known toxins, there is a variety of species with positive toxicological studies (Macías Peacok et al. 2009, Turner \& Szcawinski 1991). The plant kingdom should be considered in this sense, as a producer of poisons. Many of these compounds are restricted to certain taxa, whereas others are widely distributed, e.g., cyanogenic compounds are only found in two genera of Pinophytas and in approximately 110 families of Magnoliophyta (Nájera 1993). They are synthesized by

\section{Correspondence}

Vanesa Pérez Cuadra, Viviana Cambi, María de los Ángeles Rueda, Melina Calfuán, Laboratorio de Plantas Vasculares, Departamento de Biología, Bioquímica y Farmacia. Universidad Nacional del Sur. San Juan 670 - 8000, Bahía Blanca, Provincia de Buenos Aires, REPÚBLICA ARGENTINA.

Telephone: 542914595101 Extension: 2445. Fax: 54291 4595130.

vperezcuadra@uns.edu.ar

Ethnobotany Research \& Applications 10:077-094 (2012)

Published: April 07, 2012 
plants under certain conditions, and can be of either inorganic or organic nature. Mineral compounds are formed and accumulated in the tissues as calcium oxalate, selenium, and nitrates; organic compounds are present in the form of: alkaloids, glycosides, toxalbumins, as well as extremely complex molecules that have not yet been identified (Board et al. 2000, Nájera 1993, Szpunar 2005).

There are various hypotheses to explain the origin of toxic plant substances. They may be considered as waste products, intermediate products in metabolic processes or as secondary metabolites that fulfill non-essential functions in plants but intervene in plant/environment interactions. Some are pigments that give color to flowers and fruits, playing a fundamental role in reproduction by attracting insect pollinators or animals that contribute to seed dispersal. Others protect the plant from predators, acting as repellents due to their bitter taste and/or poisonous characteristics. They also intervene in the plants defense mechanisms against different pathogens, acting as natural pesticides. In some cases the evidence is doubtful (Olsnes 2004).

A poisonous plant is generally defined as that which contains any toxic compounds that might be harmful if consumed by humans or animals (Iramain et al. 2008, Turner \& Szcawinski 1991, Wagstaff 2008). However plants can have special structures (spines, hairs, crystals, pollen) that cause mechanical or contact injuries without being ingested and which may then result in a systemic reaction due to the chemicals liberated in the contact (Cambi \& Pérez Cuadra 2010). Both characteristics are included in a wider concept of dangerous plants (Cambi \& Pérez Cuadra 2010).

More than 700 plants species are recognized as being potentially dangerous in the world and a large number (around 500 species) of these are frequently used as ornamentals (Iramain et al. 2008). Dangerous plants are classified in two large groups according to the type of interaction with the organism: those that cause injuries by external contact and those that do so after ingestion and/ or aspiration.

Injury by contact or contact dermatitis caused by plants is known as phytodermatitis, including different types of causal agents: mechanical irritants, or chemical ones including allergens (dermatotoxic), other chemicals that do not involve the immune system (irritants) and phototoxins (dermatotoxic) (Crosby 2004, Nelson et al. 2007). Various families include species with dermatotoxic properties: Anacardiaceae (Lithrea caustica Hook. \& Arn., Mangifera indica L., Toxicodendron diversilobum (Torr. \& A. Gray) Greene), Asteraceae (Chamaemelum nobile (L.) All., Chrysanthemum morifolium Ramat., Helianthus annuus L., Taraxacum officinale F. H. Wigg.), Apiaceae (Heracleum mantegazzianum Sommier \& Levier, Pimpinella anisum L.), Fabaceae (Acacia melanoxylon R. Br., Dalbergia melanoxylon Guill. \& Perr., Dalbergia nigra (Vell.) Allemão ex Benth.), etc. Irritating plants are found in Euphorbiaceae (Euphorbia spp., Ricinus communis L.), Brassicaceae (Brassica spp.), Amaryllidaceae (Narcissus spp.), and Urticaceae (Urtica spp.) (Crosby 2004). Phytodermatitis is often observed in clinical practice, although patients do not normally consult dermatologists about minor injuries and so the exact incidence of this type of injury is unknown (Le Coz \& Ducomes 2011). Gardeners and horticulturists (occupational dermatitis) and small children are those most affected by this type of dermatitis; children represent around $20 \%$ of the total number of cases, although this percentage varies as geographic factors may make it rise to $40 \%$ (Morren \& Goossens 2011).

Poisoning by ingestion and/or aspiration is caused from the direct consumption of poisonous species, by the use of excessive (not recommended) doses of medicinal plants, for the ingestion of honey from toxic plants, smoking, inhaling smoke, eating barbecued meat supported in branches of toxic plants, etc. (Nájera 1993, Pinillos et al. 2003, Wagstaff 2008). The compounds that cause this type of intoxication are found in almost all plant families (Siener et al. 2006). In this case, there is no strict relationship with a trade, profession or age group. According to recent international reports the ingestion of plants causes between $1-2 \%$ of all poisonings, most of which are accidental and males are most affected (Macías Peacok et al. 2009). In $85 \%$ of cases the patients are children, especially those under six years with death due to ingestion of toxic plants representing $0.2 \%$ of all deaths by acute poisoning (Macías Peacok et al. 2009). Chilean statistics show that children under six years were involved in $54 \%$ of consultations concerning poisoning by plants or fungi (Botha \& Penrith 2008). In Brazil (San Pablo) the main plants responsible for poisoning (of children in particular) are $D a-$ tura sp., Ricinus sp., Manihot sp. and Dieffenbachia sp., whereas in France the main species are Arum sp., Solanum sp., Pyracantha sp., Sambucus sp. and Dieffenbachia sp. (Rocha Silva \& Takemura 2006). In the U.S.A. exposure to toxic plants is the fourth most common cause of poisoning, where $85 \%$ of exposures involve children up to four years of age (Rocha Silva \& Takemura 2006).

Injury and/or poisoning by plants can be related to the floristic composition of each region, for example, in some areas plants containing alkaloids are invasive species that cause severe intoxication or death after ingestion (Bofill et al. 2007, Stegelmeier et al. 1999). In numerous parts of the world consumption of food plants which have specific toxic compounds in very low quantities (such as calcium oxalate) is common but this concentration can rise to a very high levels under certain environmental conditions causing health problems (Noonon \& Savage 1999).

The most frequent symptoms presented on exposure to a dangerous plant are: digestive or neurological problems or skin reactions. Clinical cases are often not easy 


\section{Pérez Cuadra et al. - Consequences of the Loss of Traditional Knowledge: The risk of injurious and toxic plants growing in kindergartens}

to solve, they depend on good diagnosis (Botha \& Penrith 2008, Nájera 1993). Unlike other types of poisoning those caused by plants are usually difficult to diagnose as, in general, people do not admit to have ingested a plant and doctors have very little botanical knowledge, so identification of the plant and then treatment of the potential toxin is usually difficult (Macías Peacok et al. 2009).

The prevention of injuries and/or poisoning by dangerous plants has been studied in greater detail in the U.S.A., Canada, Australia and New Zealand. They have formed specialized emergency services and also developed programs of dissemination and public awareness about the care that should be taken with plants in private homes, educational institutions, public places, etc. They have also prepared lists of plants that are appropriate, or not recommended, for landscaping places with different purposes (children's playgrounds, dog walking, etc.). In Latin America, in particular, some countries like Nicaragua include the prevention of poisoning by plants in general safety campaigns, as another potentially dangerous toxic agent. In the South American countries, such as Argentina, there are some local alerts on the danger of certain species, but without giving any great importance to the issue. However, in the same country and unlike the case of humans, there are several studies on toxic plants that affect cattle and other domesticated animals, since in the case of cattle it is considered important for the economic impact on the country (Freire et al. 2005, Gallo 1987, Robles et al. 2000), whereas in the case of pets, it has always been a topic of intense research by veterinarians due to the incidence of poisoning of young animals (Iramain et al. 2008, Zeinsteger \& Gurni 2004). In the partido of Bahía Blanca there are no previous reports on any issue concerning toxic plants, there being a general lack of knowledge on the importance of the subject.

The absence of a national program of diffusion not only about the existence of dangerous plants but also about the cares the population needs to take with them is total in Argentina. Due to this failing, the aim of this study is to evaluate the presence of dangerous ornamental plants in kindergartens and child care centers under the hypothesis that they are present in these institutions (where children spend several hours a day), and that the teachers in the majority do not know about their existence. This last point has been studied through interviews with teachers in charge of each Institution, initiating a way for mutual interaction that would allow them to understand the importance that urban people give to the plants that surround them.

\section{Materials and methods}

The partido of Bahía Blanca is in the southwest of the province of Buenos Aires, $700 \mathrm{~km}$ south of Buenos Aires City, capital of Argentina. It consists of the capital city Bahía Blanca $\left(38^{\circ} 43^{\prime} 2^{\prime \prime} \mathrm{S}\right.$ and $\left.62^{\circ} 15^{\prime} 54^{\prime \prime} \mathrm{W}\right)$ and the towns of Ingeniero White, General Daniel Cerri, and Cabildo. There are 284,776 inhabitants in the partido, of which 20,993 are children between 0-5 years and of these 9,425 (INDEC 2001) attend one of the 85 public and private kindergartens and child care centers recognized by the Dirección General de Cultura y Educación of Buenos Aires province in 2009.

Floristic evaluation of all these educational institutions was carried out between August 2009 and July 2010 using ad-hoc inventory forms to collect the following data: name of species found, number of plants, type of habitat (inside or outside; in the ground or in flower pots), probability of contact, and specific area where they are located (Cambi \& Pérez Cuadra 2010). These forms were tried out in a pilot sampling at a small number of the educational institutions. After this first experience, they were adjusted so that appropriate data could be collected in all institutions.

A specific bibliography on cultivated ornamental plants was consulted to identification the different plant species found (Armitage 2004, 2011, Austin 2005, Bryan 2002; Dimitri 1988, Dirr 2002, Hurrell 2006, 2007, Innes \& Glass 1997, Llamas 2003, Sajeva \& Constanzo 2001, Schmid 2002, Zachos 2008). Specimens were preserved and deposited in the Herbarium of the Departamento de Biología, Bioquímica y Farmacia of the Universidad Nacional del Sur (BBB). The scientific names used and their taxonomic position were updated following the international database Tropicos.org (2011).

In each visit, the teacher in charge of the kindergarten was interviewed (85 teachers in total) to find out: whether the property had been designed as a kindergarten, if the ornamental plants had been there since its inauguration, if there had been any planned landscaping of the site, if they have had any problems with poisonous plants, whether they knew about dangerous ornamentals, whether they were interested in information on this topic and if they would be interested in some form of labelling of the dangerous plants (Cambi \& Pérez Cuadra 2010).

In order to conduct this work, authorizations were processed at the Jefatura Distrital de Educación of Bahía Blanca City, which endorsed the study and allowed the collection of all necessary data. In the particular case of the interviews, teachers were consulted about their willingness to answer the questionnaire, assuming the confidentially of the information provided.

The ornamental plant data and the information collected in the interviews were summarized in general forms to make a data base of the most relevant information. The ornamental plants were first divided into harmless and dangerous groups. The latter were further classified according to the categories cited by Nelson et al. (2007) and Wagstaff (2008), adding a category for respiratory diseas- 
es that was not considered by those authors. The following classes were included in the study: harmless (plants for which there are no current bibliographical records to indicate that they cause any health hazards); moderately dangerous (those that do not cause serious health problems, e.g., they may cause respiratory diseases and phytodermatitis or contain calcium oxalate crystals and gastrointestinal toxins); and highly dangerous (those that may cause serious health problems) which includes those containing alkaloids, anticholinergic poisons, cardioactive steroids or cardiac glycosides, convulsant poisons, cyanogenic compounds, mitotic inhibitors, sodium channel activators and toxalbumins).

Table 1. Harmless ornamental species found in kindergartens of Bahía Blanca City (Argentina).

\begin{tabular}{|c|c|}
\hline Classification & Ornamental Species \\
\hline \multicolumn{2}{|c|}{ Subclass Polypodiidae } \\
\hline Aspleniaceae & Asplenium nidus $\mathrm{L}$. \\
\hline Davalliaceae & $\begin{array}{l}\text { Nephrolepis cordifolia } \\
\text { (L.) C. Presl }\end{array}$ \\
\hline \multicolumn{2}{|c|}{ Subclass Pinidae } \\
\hline Cupressaceae & Thuja sp. \\
\hline \multirow[t]{2}{*}{ Pinaceae } & Picea sp. \\
\hline & Pinus sp. \\
\hline \multicolumn{2}{|c|}{ Subclass Magnoliidae, Superorder Asteranae } \\
\hline \multirow[t]{2}{*}{ Acanthaceae } & Acanthus mollis L. \\
\hline & Beloperone guttata Brandegee \\
\hline Adoxaceae & Viburnum tinus $\mathrm{L}$. \\
\hline \multirow[t]{3}{*}{ Apiaceae } & Apium graveolens $\mathrm{L}$. \\
\hline & Daucus carota L. \\
\hline & Petroselinum crispum (Mill.) Fuss \\
\hline \multirow[t]{5}{*}{ Apocynaceae } & Araujia hortorum E. Fourn. \\
\hline & Araujia sericifera Brot. \\
\hline & Stapelia variegata $\mathrm{L}$. \\
\hline & $\begin{array}{l}\text { Trachelospermum } \\
\text { jasminoides (Lindl.) Lem. }\end{array}$ \\
\hline & Vinca major $\mathrm{L}$. \\
\hline \multirow[t]{8}{*}{ Asteraceae } & Bellis perennis L. \\
\hline & $\begin{array}{l}\text { Chrysanthemum } \\
\text { leucanthemum L. }\end{array}$ \\
\hline & Cichorium intybus L. \\
\hline & Euryops pectinatus (L.) Cass. \\
\hline & Farfugium japonicum (L.) Kitam. \\
\hline & Felicia amelloides (L.) Voss \\
\hline & Gazania splendens Lem. \\
\hline & Gerbera sp. \\
\hline
\end{tabular}

It should be clarified that some species were not at an appropriate phenological stage for their specific identification and so they were estimated in number, but not assigned to a special category, so as not to overestimate any of the categories.

\section{Results}

In the 85 kindergartens and child care centers, 312 ornamental species were recorded of which 303 were identified taxonomically; nine were not at an appropriate phenological stage at the time of the census so could not be identified. Of the total number of identified species, 208 $(69 \%)$ are considered harmless (Table 1$)$, whereas the

\begin{tabular}{|c|c|}
\hline Classification & Ornamental Species \\
\hline \multirow[t]{7}{*}{ Asteraceae } & Helianthus tuberosus L. \\
\hline & Lactuca sativa L. \\
\hline & Osteospermum fruticosum Norl. \\
\hline & Senecio angulatus L.f. \\
\hline & Senecio cineraria DC. \\
\hline & $\begin{array}{l}\text { Senecio mikanioides } \\
\text { Otto ex Walp. }\end{array}$ \\
\hline & Zinnia sp. \\
\hline Balsaminaceae & Impatiens balsamina L. \\
\hline \multirow[t]{6}{*}{ Bignoniaceae } & Campsis radicans (L.) Bureau \\
\hline & Catalpa bignonioides Walter \\
\hline & $\begin{array}{l}\text { Jacaranda jasminoides } \\
\text { (Thunb.) Sandwith }\end{array}$ \\
\hline & Jacaranda mimosifolia D. Don \\
\hline & $\begin{array}{l}\text { Podranea ricasoliana } \\
\text { (Tanfani) Sprague }\end{array}$ \\
\hline & Tabebuia sp. \\
\hline Campanulaceae & Lobelia erinus L. \\
\hline Caprifoliaceae & $\begin{array}{l}\text { Abelia x grandiflora (Rovelli } \\
\text { ex André) Rehder }\end{array}$ \\
\hline \multirow[t]{2}{*}{ Convolvulaceae } & Convolvulus bonariensis Cav. \\
\hline & Dichondra sp. \\
\hline Hydrangeaceae & Philadelphus coronarius L. \\
\hline \multirow[t]{6}{*}{ Lamiaceae } & Coleus blumei Benth. \\
\hline & Leonotis leonurus (L.) R. Br. \\
\hline & Plectranthus australis R. Br. \\
\hline & $\begin{array}{l}\text { Plectranthus ciliatus } \\
\text { E. Mey. ex Benth. }\end{array}$ \\
\hline & Rosmarinus officinalis L. \\
\hline & Salvia officinalis L. \\
\hline
\end{tabular}


Pérez Cuadra et al. - Consequences of the Loss of Traditional Knowledge:
The risk of injurious and toxic plants growing in kindergartens

\begin{tabular}{|c|c|}
\hline Classification & Ornamental Species \\
\hline \multirow[t]{2}{*}{ Lamiaceae } & $\begin{array}{l}\text { Salvia splendens Sellow } \\
\text { ex Wied-Neuw. }\end{array}$ \\
\hline & Teucrium fruticans L. \\
\hline \multirow[t]{5}{*}{ Oleaceae } & Fraxinus sp. \\
\hline & Jasminum mesnyi Hance \\
\hline & Jasminum polyanthum Franch. \\
\hline & Ligustrum sinense Lour. \\
\hline & Syringa vulgaris $\mathrm{L}$. \\
\hline \multirow[t]{2}{*}{ Plttosporaceae } & $\begin{array}{l}\text { Pittosporum tenuifolium } \\
\text { Banks \& Sol. ex Gaertn. }\end{array}$ \\
\hline & $\begin{array}{l}\text { Pittosporum tobira } \\
\text { (Trunb.) W. T. Aiton }\end{array}$ \\
\hline \multirow[t]{2}{*}{ Plantaginaceae } & Antirrhinum majus L. \\
\hline & Linaria texana Scheele \\
\hline Primulaceae & Cyclamen persicum Mill. \\
\hline Scrophulariaceae & Buddleja davidii Franch. \\
\hline \multirow[t]{2}{*}{ Solanaceae } & Petunia hybrida Vilm. \\
\hline & Solanum crispum Ruiz \& Pav. \\
\hline Theaceae & Camellia japonica L. \\
\hline \multirow[t]{2}{*}{ Verbenaceae } & Aloysia citrodora Paláu \\
\hline & Duranta sp. \\
\hline \multicolumn{2}{|c|}{ Subclass Magnoliidae, Superorder Buxanae } \\
\hline Buxaceae & Buxus sempervirens L. \\
\hline \multicolumn{2}{|c|}{ Subclass Magnoliidae, Superorder Caryophyllanae } \\
\hline \multirow[t]{4}{*}{ Aizoaceae } & $\begin{array}{l}\text { Aptenia cordifolia (L.f.) } \\
\text { Schwantes }\end{array}$ \\
\hline & $\begin{array}{l}\text { Drosanthemum floribundum } \\
\text { (Haw.) Schwantes }\end{array}$ \\
\hline & $\begin{array}{l}\text { Glottiphyllum oligocarpum } \\
\text { L. Bolus }\end{array}$ \\
\hline & $\begin{array}{l}\text { Lampranthus spectabilis } \\
\text { (Haw.) N.E. Br. }\end{array}$ \\
\hline \multirow[t]{3}{*}{ Amaranthaceae } & Beta vulgaris $\mathrm{L}$. \\
\hline & $\begin{array}{l}\text { Beta vulgaris L. var. cicla } \\
\text { (L.) W.D.J. Koch }\end{array}$ \\
\hline & Spinacia oleracea L. \\
\hline \multirow[t]{3}{*}{ Cactaceae } & $\begin{array}{l}\text { Schlumbergera russelianum } \\
\text { (Hook.) Britton \& Rose }\end{array}$ \\
\hline & $\begin{array}{l}\text { Schulembergera truncata } \\
\text { (Haw.) Moran }\end{array}$ \\
\hline & $\begin{array}{l}\text { Trichocereus candicans (K. } \\
\text { Schum.) Britton \& Rose }\end{array}$ \\
\hline Didiereaceae & Portulacaria afra Jacq. \\
\hline \multirow[t]{2}{*}{ Nyctaginaceae } & Bougainvillea spectabilis Willd. \\
\hline & Mirabilis jalapa L. \\
\hline
\end{tabular}

\begin{tabular}{|c|c|}
\hline Classification & Ornamental Species \\
\hline Plumbaginaceae & Plumbago auriculata Lam. \\
\hline Talinaceae & $\begin{array}{l}\text { Talinum paniculatum } \\
\text { (Jacq.) Gaertn. }\end{array}$ \\
\hline Tamaricaceae & Tamarix gallica L. \\
\hline \multicolumn{2}{|c|}{ Subclass Magnoliidae, Superorder Lilianae } \\
\hline \multirow[t]{5}{*}{ Amaryllidaceae } & $\begin{array}{l}\text { Agapanthus africanus } \\
\text { (L.) Hoffmanns. }\end{array}$ \\
\hline & Allium triquetrum $\mathrm{L}$. \\
\hline & Crinum x powellii hort. ex Baker \\
\hline & Ipheion uniflorum Raf. \\
\hline & $\begin{array}{l}\text { Nothoscordum inodorum } \\
\text { (Aiton) Asch. \& Graebn. }\end{array}$ \\
\hline Araceae & Aglaonema commutatum Schott \\
\hline \multirow[t]{5}{*}{ Arecaceae } & Chamaedorea sp. \\
\hline & Chamaerops humilis L. \\
\hline & Rhapis excelsa (Trunb.) A. Henry \\
\hline & Trachycarpus sp. \\
\hline & $\begin{array}{l}\text { Washingtonia filifera (Linden ex } \\
\text { André) } \mathrm{H} \text {. Wendl. ex de Bary }\end{array}$ \\
\hline \multirow[t]{14}{*}{ Asparagaceae } & $\begin{array}{l}\text { Asparagus asparagoides } \\
\text { (L.) Druce }\end{array}$ \\
\hline & $\begin{array}{l}\text { Asparagus setaceus } \\
\text { (Kunth) Jessop }\end{array}$ \\
\hline & Asparagus sprengeri Regel \\
\hline & Aspidistra elatior Blume \\
\hline & $\begin{array}{l}\text { Chlorophytum comosum } \\
\text { (Trunb.) Jacques }\end{array}$ \\
\hline & Cordyline australis Hook.f. \\
\hline & Dracaena deremensis Engl. \\
\hline & Dracaena fragrans (L.) Ker Gawl. \\
\hline & Dracaena marginata Lam. \\
\hline & $\begin{array}{l}\text { Drimiopsis maculata } \\
\text { Lindl. \& J. Paxton }\end{array}$ \\
\hline & Ornithogalum caudatum Aiton \\
\hline & Ruscus hypoglossum L. \\
\hline & Sansevieria trifasciata Prain \\
\hline & Yucca filamentosa $\mathrm{L}$. \\
\hline \multirow[t]{3}{*}{ Bromeliaceae } & Aechmea fasciata (Lindl.) Baker \\
\hline & Cryptanthus acaulis (Lindl.) Beer \\
\hline & Tillandsia sp. \\
\hline Cannaceae & Canna indica L. \\
\hline \multirow[t]{2}{*}{ Commelinaceae } & Commelina erecta L. \\
\hline & $\begin{array}{l}\text { Tradescantia pallida } \\
\text { (Rose) D.R. Hunt }\end{array}$ \\
\hline
\end{tabular}




\begin{tabular}{|c|c|}
\hline Classification & Ornamental Species \\
\hline Cyperaceae & Cyperus alternifolius L. \\
\hline Marantaceae & Maranta leuconeura E. Morren \\
\hline \multirow[t]{3}{*}{ Xanthorrhoeaceae } & Gasteria sp. \\
\hline & Haworthia fasciata Haw. \\
\hline & $\begin{array}{l}\text { Phormium tenax J.R. } \\
\text { Forst. \& G. Forst. }\end{array}$ \\
\hline \multicolumn{2}{|c|}{ Subclass Magnoliidae, Superorder Magnolianae } \\
\hline Magnoliaceae & Magnolia grandiflora L. \\
\hline \multicolumn{2}{|c|}{ Subclass Magnoliidae, Superorder Myrothamnanae } \\
\hline Altinginaceae & Liquidambar styraciflua L. \\
\hline \multirow[t]{15}{*}{ Crassulaceae } & $\begin{array}{l}\text { Aeonium arboreum (L.) } \\
\text { Webb \& Berthel. }\end{array}$ \\
\hline & Aeonium gomerense Praeger \\
\hline & Crassula arborescens Willd. \\
\hline & Crassula brevifolia Harv. \\
\hline & Crassula multicava Lem. \\
\hline & Crassula ovata (Mill.) Druce \\
\hline & $\begin{array}{l}\text { Crassula subacaulis } \\
\text { subsp. erosula Toelken }\end{array}$ \\
\hline & Echeveria sp. \\
\hline & Sedum clavatum R.T. Clausen \\
\hline & $\begin{array}{l}\text { Sedum dendroideum } \\
\text { Moc. \& Sessé ex DC. }\end{array}$ \\
\hline & Sedum lucidum R.T. Clausen \\
\hline & Sedum pachyphyllum Rose \\
\hline & Sedum palmeri S. Watson \\
\hline & Sedum rupestre L. \\
\hline & Sedum telephium $\mathrm{L}$. \\
\hline \multicolumn{2}{|c|}{ Subclass Magnoliidae, Superorder Ranunculanae } \\
\hline Berberidaceae & Nandina domestica Thunb. \\
\hline \multirow[t]{2}{*}{ Ranunculaceae } & Aquilegia columbiana Rydb. \\
\hline & Ranunculus asiaticus L. \\
\hline \multicolumn{2}{|c|}{ Subclass Magnoliidae, Superorder Rosanae } \\
\hline Anacardiaceae & Schinus molle L. \\
\hline \multirow[t]{3}{*}{ Begoniaceae } & Begonia aconitifolia DC. \\
\hline & Begonia rex Putz. \\
\hline & $\begin{array}{l}\text { Begonia semperflorens } \\
\text { Link \& Otto }\end{array}$ \\
\hline \multirow[t]{3}{*}{ Brassicaceae } & Lobularia maritima (L.) Desv. \\
\hline & Matthiola incana (L.) W. T. Aiton \\
\hline & Raphanus sativus L. \\
\hline Casuarinaceae & Casuarina cunninghamiana Miq. \\
\hline Celastraceae & Euonymus japonicus Thunb. \\
\hline
\end{tabular}

\begin{tabular}{|c|c|}
\hline Classification & Ornamental Species \\
\hline \multirow[t]{2}{*}{ Eleagnaceae } & Eleagnus angustifolia L. \\
\hline & Eleagnus pungens Trunb. \\
\hline Euphorbiaceae & Manihot flabellifolia Pohl. \\
\hline \multirow[t]{12}{*}{ Fabaceae } & Acacia baileyana F. Muell. \\
\hline & Acacia dealbata Link \\
\hline & Acacia retinodes Schltdl. \\
\hline & Acacia visco Lorentz ex Griseb. \\
\hline & Albizia julibrissin Durazz. \\
\hline & $\begin{array}{l}\text { Caesalpinia gilliesii (Wall. } \\
\text { ex Hook.) D. Dietr. }\end{array}$ \\
\hline & Erythrina crista-galli L. \\
\hline & Pisum sativum $\mathrm{L}$. \\
\hline & Prosopis alba Griseb. \\
\hline & Sophora japonica L. \\
\hline & Spartium junceum L. \\
\hline & Trifolium pratense L. \\
\hline Geraniaceae & Geranium molle L. \\
\hline Juglandaceae & Juglans regia L. \\
\hline Linaceae & Linum usitatissimum L. \\
\hline \multirow[t]{3}{*}{ Lythraceae } & Cuphea hyssopifolia Kunth \\
\hline & Lagerstroemia indica L. \\
\hline & Punica granatum L. \\
\hline \multirow[t]{7}{*}{ Malvaceae } & $\begin{array}{l}\text { Abutilon pictum (Gillies } \\
\text { ex Hook. Arn.) Walp. }\end{array}$ \\
\hline & $\begin{array}{l}\text { Brachychiton populneus } \\
\text { (Schott \& Endl.) R. Br. }\end{array}$ \\
\hline & Chorisia speciosa A. St.-Hil. \\
\hline & Cotoneaster franchetii Bois \\
\hline & $\begin{array}{l}\text { Cotoneaster glaucophyllus } \\
\text { Franch. }\end{array}$ \\
\hline & Hibiscus syriacus L. \\
\hline & Lavatera maritima Gouan \\
\hline \multirow[t]{2}{*}{ Moraceae } & Ficus elastica Roxb. ex Hornem. \\
\hline & Morus sp. \\
\hline \multirow[t]{2}{*}{ Myrtaceae } & Callistemon rigidus $\mathrm{R}$. Br. \\
\hline & Eucalyptus sp. \\
\hline \multirow[t]{2}{*}{ Onagraceae } & Fuchsia hybrida hort. ex V. Vilm. \\
\hline & $\begin{array}{l}\text { Godetia amoena } \\
\text { (Lenhm.) G. Don }\end{array}$ \\
\hline Oxalidaceae & Oxalis sp. \\
\hline Passifloraceae & Passiflora caerulea L. \\
\hline Rosaceae & $\begin{array}{l}\text { Chaenomeles japonica } \\
\text { (Thunb.) Lindl. }\end{array}$ \\
\hline
\end{tabular}


Pérez Cuadra et al. - Consequences of the Loss of Traditional Knowledge:
The risk of injurious and toxic plants growing in kindergartens

\begin{tabular}{|l|l|}
\hline Classification & Ornamental Species \\
\hline Rosaceae & Cydonia oblonga Mill. \\
\cline { 2 - 2 } & Fragaria sp. \\
\cline { 2 - 2 } & Malus sp. \\
\cline { 2 - 2 } & Photinia sp. \\
\cline { 2 - 2 } & Pyracantha sp. \\
\cline { 2 - 2 } & Pyrus communis L. \\
\cline { 2 - 2 } & Rosa sp. \\
\cline { 2 - 2 } & Spiraea cantoniensis Lour. \\
\hline Salicaceae & Populus alba L. \\
\cline { 2 - 2 } & Populus nigra L. \\
\cline { 2 - 2 } & Salix babylonica L. \\
\cline { 2 - 2 } & Salix sp. \\
\hline
\end{tabular}

\begin{tabular}{|l|l|}
\hline Classification & Ornamental Species \\
\hline Tropaeolaceae & Tropaeolum majus L. \\
\hline Ulmaceae & Ulmus minor Mill. \\
\hline Urticaceae & Parietaria officinalis L. \\
\cline { 2 - 2 } & $\begin{array}{l}\text { Pilea cadierei Gagnep. } \\
\text { \& Guillaumin }\end{array}$ \\
\cline { 2 - 2 } & Pilea nummulariifolia (Sw.) Wedd. \\
\cline { 2 - 2 } & Soleirolia soleirolii (Req.) Dandy \\
\hline Violaceae & Viola tricolor L. \\
\cline { 2 - 2 } & Viola odorata L. \\
\hline Vitaceae & $\begin{array}{l}\text { Parthenocissus quinquefolia } \\
\text { (L.) Planch. }\end{array}$ \\
\cline { 2 - 2 } & $\begin{array}{l}\text { Parthenocissus tricuspidata } \\
\text { (Siebold \& Zucc.) Planch. }\end{array}$ \\
\hline
\end{tabular}

other 95 species $(31 \%)$ might produce some kind of injury (Tables 2 and 3). Among the latter group, 66 species

$(70 \%)$ are considered moderately harmful (Table 2$)$ and 29 species $(30 \%)$ are highly dangerous (Table 3$)$.

Table 2. Moderately dangerous ornamental species growing in kindergartens of Bahía Blanca City (Argentina), with details of the toxic part and their specific category of toxicity.

\begin{tabular}{|c|c|c|c|}
\hline Classification & Ornamental species & Toxic part & Specific category \\
\hline \multicolumn{4}{|c|}{ Subclass Pinidae } \\
\hline \multirow[t]{3}{*}{ Cupressaceae } & Calocedrus decurrens (Torr.) Florin & Pollen & \multirow[t]{2}{*}{ Respiratory ailments } \\
\hline & Cupressus sp. & Pollen & \\
\hline & Juniperus sp. & Leaves & Phytodermatitis \\
\hline Pinaceae & Cedrus sp. & Pollen & Respiratory ailments \\
\hline \multicolumn{4}{|c|}{ Subclass Magnoliidae, Superorder Asteranae } \\
\hline \multirow[t]{2}{*}{ Araliaceae } & Hedera helix L. & Leaves and fruits & Phytodermatitis \\
\hline & Schefflera digitata J. R. Forst. \& G. Forst. & Leaves & $\begin{array}{l}\text { Calcium oxalate } \\
\text { crystals }\end{array}$ \\
\hline \multirow[t]{6}{*}{ Asteraceae } & Argyranthemum frutescens (L.) Sch. Bip. & Leaves and stems & \multirow[t]{6}{*}{ Phytodermatitis } \\
\hline & Calendula officinalis L. & Leaves & \\
\hline & Chrysanthemum sp. & Flowers & \\
\hline & Tagetes patula L. & Leaves & \\
\hline & Taraxacum officinale F.H. Wigg. & Leaves & \\
\hline & Wedelia glauca (Ortega) O. Hoffm. ex Hicken & All plant & \\
\hline Caprifoliaceae & Lonicera japonica Trunb. & Fruits & Gastrointestinal toxins \\
\hline Garryaceae & Aucuba japonica Trunb. & All plant & Gastrointestinal toxins \\
\hline \multirow[t]{3}{*}{ Lamiaceae } & Lavandula officinalis Chaix & Leaves & \multirow[t]{3}{*}{ Phytodermatitis } \\
\hline & Mentha sp. & Leaves & \\
\hline & Plectranthus coleoides Benth. & Leaves & \\
\hline \multirow[t]{2}{*}{ Oleaceae } & Ligustrum lucidum W.T. Aiton & All plant & Gastrointestinal toxins \\
\hline & Olea europaea L. & Leaves & Phytodermatitis \\
\hline \multirow[t]{3}{*}{ Primulaceae } & Primula malacoides Franch. & Leaves & \multirow[t]{3}{*}{ Phytodermatitis } \\
\hline & Primula obconica Hance & Leaves & \\
\hline & Primula veris $\mathrm{L}$. & & \\
\hline
\end{tabular}




\begin{tabular}{|c|c|c|c|}
\hline Classification & Ornamental species & Toxic part & Specific category \\
\hline Rubiaceae & Gardenia jasminoides J. Ellis & Fruits & Phytodermatitis \\
\hline Scrophulariaceae & Myoporum laetum G. Forst. & Leaves and fruits & Gastrointestinal toxins \\
\hline Solanaceae & Lycopersicon esculentum Mill. & Leaves & Phytodermatitis \\
\hline Verbenaceae & Lantana camara L. & Leaves and fruits & Gastrointestinal toxins \\
\hline \multicolumn{4}{|c|}{ Subclass Magnoliidae, Superorder Caryophyllanae } \\
\hline Cactaceae & Opuntia sp. & Spines & Phytodermatitis \\
\hline Caryophyllaceae & Dianthus plumarius $\mathrm{L}$. & All plant & Phytodermatitis \\
\hline Phytolaccaceae & Phytolacca dioica L. & Leaves and roots & Gastrointestinal toxins \\
\hline Portulacaceae & Portulaca grandiflora Hook. & Leaves & $\begin{array}{l}\text { Calcium oxalate } \\
\text { crystals }\end{array}$ \\
\hline \multicolumn{4}{|c|}{ Subclass Magnoliidae, Superorder Lilianae } \\
\hline \multirow[t]{2}{*}{ Amaryllidaceae } & Allium cepa L. & Bulbs & Phytodermatitis \\
\hline & Narcissus pseudonarcissus L. & Bulbs & Phytodermatitis \\
\hline \multirow[t]{10}{*}{ Araceae } & Alocasia sp. & Leaves and stems & \multirow{8}{*}{$\begin{array}{l}\text { Calcium oxalate } \\
\text { crystals }\end{array}$} \\
\hline & Arum italicum Mill. & All plant & \\
\hline & Caladium bicolor Vent. & All plant & \\
\hline & Dieffenbachia maculata (Lood.) G. Don & All plant & \\
\hline & Monstera deliciosa Liebm. & Leaves & \\
\hline & Philodendron sp. & Leaves & \\
\hline & $\begin{array}{l}\text { Scindapsus aureus (Linden \& } \\
\text { André) Engl. \& K. Krause }\end{array}$ & Leaves & \\
\hline & Spathiphyllum wallisii Regel & Leaves & \\
\hline & Syngonium podophyllum Schott & Leaves & \multirow{2}{*}{$\begin{array}{l}\text { Calcium oxalate } \\
\text { crystals }\end{array}$} \\
\hline & Zantedeschia aethiopica (L.) Spreg. & Leaves & \\
\hline Asparagaceae & Agave americana L. & Leaves & Phytodermatitis \\
\hline Commelinaceae & Tradescantia fluminensis Vell. & Leaves and stems & Phytodermatitis \\
\hline Iridaceae & Iris germanica L. & Leaves and rhizomes & Gastrointestinal toxins \\
\hline Liliaceae & Lilium sp. & All plant & Gastrointestinal toxins \\
\hline \multirow[t]{3}{*}{ Xanthorrhoeaceae } & Aloe arborescens Mill. & Leaves & \multirow[t]{3}{*}{ Gastrointestinal toxins } \\
\hline & Aloe aristata Haw. & Leaves & \\
\hline & Aloe saponaria Haw. & Leaves & \\
\hline \multicolumn{4}{|c|}{ Subclass Magnoliidae, Superorder Magnolianae } \\
\hline Lauraceae & Laurus nobilis L. & Leaves & Phytodermatitis \\
\hline \multicolumn{4}{|c|}{ Subclass Magnoliidae, Superorder Proteanae } \\
\hline Platanaceae & Platanus acerifolia (Aiton) Willd. & Fruits & Respiratory ailments \\
\hline \multicolumn{4}{|c|}{ Subclass Magnoliidae, Superorder Rosanae } \\
\hline Euphorbiaceae & Euphorbia peplus L. & All plant & Phytodermatitis \\
\hline \multirow[t]{2}{*}{ Fabaceae } & Acacia melanoxylon $\mathrm{R}$. Br. & Pollen & Phytodermatitis \\
\hline & Phaseolus vulgaris $\mathrm{L}$. & Seeds & Gastrointestinal toxins \\
\hline Fagaceae & Quercus robur L. & Seeds & Gastrointestinal toxins \\
\hline Geraniaceae & Pelargonium sp. & Leaves and stems & Phytodermatitis \\
\hline Malvaceae & Hibiscus rosa-sinensis L. & Leaves & Phytodermatitis \\
\hline
\end{tabular}


Pérez Cuadra et al. - Consequences of the Loss of Traditional Knowledge:
The risk of injurious and toxic plants growing in kindergartens

\begin{tabular}{|c|c|c|c|}
\hline Classification & Ornamental species & Toxic part & Specific category \\
\hline \multirow[t]{2}{*}{ Moraceae } & Ficus benjamina L. & All plant & \multirow[t]{7}{*}{ Phytodermatitis } \\
\hline & Ficus carica L. & All plant & \\
\hline \multirow[t]{5}{*}{ Rutaceae } & Citrus aurantium L. & Leaves & \\
\hline & Citrus maxima (Burm.) Merr. & Leaves & \\
\hline & Citrus limon (L.) Osbeck & Leaves & \\
\hline & Citrus sinensis (L.) Osbeck & Leaves & \\
\hline & Ruta graveolens L. & Leaves & \\
\hline Simaroubaceae & Ailanthus altissima (Mill.) Swingle & All plant & Respiratory ailments \\
\hline Vitaceae & \begin{tabular}{|l} 
Vitis vinifera L. \\
Vine
\end{tabular} & Fruits & Gastrointestinal toxins \\
\hline
\end{tabular}

Table 3. Highly dangerous ornamental species growing in kindergartens of Bahía Blanca City (Argentina), with details of the toxic part and their specific category of toxicity.

\begin{tabular}{|c|c|c|c|}
\hline Family & Ornamental species & Toxic part & Specific category \\
\hline \multicolumn{4}{|c|}{ Subclass Magnoliidae, Superorder Asteranae } \\
\hline \multirow[t]{2}{*}{ Apocynaceae } & Nerium oleander L. & All plant & $\begin{array}{l}\text { Cardioactive steroids } \\
\text { or cardiac glycosides }\end{array}$ \\
\hline & Vinca rosea L. & All plant & Mitotic inhibitors \\
\hline Asteraceae & Senecio madagascariensis Poir. & All plant & Alkaloids \\
\hline Convolvulaceae & Ipomoea batatas (L.) Lam. & Roots & Alkaloids \\
\hline Hydrangeaceae & Hydrangea macrophylla (Thunb.) Ser. & All plant & $\begin{array}{l}\text { Cyanogenic } \\
\text { compounds }\end{array}$ \\
\hline \multirow[t]{3}{*}{ Solanaceae } & Brunfelsia australis Benth. & All plant & \multirow[t]{3}{*}{ Alkaloids } \\
\hline & Nicotiana glauca Graham & All plant & \\
\hline & Solanum tuberosum L. & Tubers & \\
\hline \multicolumn{4}{|c|}{ Subclass Magnoliidae, Superorder Lilianae } \\
\hline Amaryllidaceae & Clivia miniata Regel & All plant & Alkaloids \\
\hline \multicolumn{4}{|c|}{ Subclass Magnoliidae, Superorder Magnolianae } \\
\hline Lauraceae & Persea americana Mill. & Leaves & $\begin{array}{l}\text { Cardioactive steroids } \\
\text { or cardiac glycosides }\end{array}$ \\
\hline \multicolumn{4}{|c|}{ Subclass Magnoliidae, Superorder Myrothamnanae } \\
\hline \multirow[t]{3}{*}{ Crassulaceae } & Kalanchoe blossfeldiana Poelln. & All plant & \multirow{3}{*}{$\begin{array}{l}\text { Cardioactive steroids } \\
\text { or cardiac glycosides }\end{array}$} \\
\hline & $\begin{array}{l}\text { Kalanchoe daigremontiana Raym.-Hamet. } \\
\text { \& H. Perrier }\end{array}$ & All plant & \\
\hline & $\begin{array}{l}\text { Kalanchoe fedtschenkoi Raym.- } \\
\text { Hamet. \& H. Perrier }\end{array}$ & All plant & \\
\hline \multicolumn{4}{|c|}{ Subclass Magnoliidae, Superorder Ranunculanae } \\
\hline Papaveraceae & Papaver sp. & All plant & Alkaloids \\
\hline \multicolumn{4}{|c|}{ Subclass Magnoliidae, Superorder Rosanae } \\
\hline Euphorbiaceae & Ricinus communis L. & All plant & Toxalbumins \\
\hline \multirow[t]{3}{*}{ Fabaceae } & Lathyrus latifolius L. & Seeds & Alkaloids \\
\hline & Robinia pseudoacacia L. & $\begin{array}{l}\text { Leaves, bark } \\
\text { and seeds }\end{array}$ & \multirow[t]{2}{*}{ Toxalbumins } \\
\hline & $\begin{array}{l}\text { Robinia pseudaocacia L. var. } \\
\text { umbraculifera DC. }\end{array}$ & Leaves and bark & \\
\hline
\end{tabular}




\begin{tabular}{|c|c|c|c|}
\hline Family & Ornamental species & Toxic part & Specific category \\
\hline \multirow[t]{2}{*}{ Fabaceae } & Robinia pseudoacacia L. "casque rouge" & $\begin{array}{l}\text { Leaves, bark } \\
\text { and seeds }\end{array}$ & Toxalbumins \\
\hline & Wisteria sinensis (Sims) Sweet & All plant & $\begin{array}{l}\text { Cardioactive steroids } \\
\text { or cardiac glycosides }\end{array}$ \\
\hline Meliaceae & Melia azedarach L. & Fruits and bark & Alkaloids \\
\hline \multirow[t]{8}{*}{ Rosaceae } & Eriobotrya japonica (Thunb.) Lindl. & Fruits and seeds & \multirow{8}{*}{$\begin{array}{l}\text { Cyanogenic } \\
\text { compounds }\end{array}$} \\
\hline & Prunus amygdalus Batsch & Seeds & \\
\hline & Prunus armeniaca L. & Seeds & \\
\hline & Prunus avium (L.) L. & Seeds & \\
\hline & Prunus cerasifera Ehrh. & Seeds & \\
\hline & Prunus domestica L. & Seeds & \\
\hline & Prunus persica (L.) Batsch & Seeds & \\
\hline & $\begin{array}{l}\text { Prunus persica (L.) Batsch } \\
\text { var. nectarina Maxim. }\end{array}$ & Seeds & \\
\hline
\end{tabular}

Of the moderately harmful species, 35 (54\%) produce phytodermatitis, $14(21 \%)$ contain gastrointestinal toxins, $12(18 \%)$ possess calcium oxalate crystals in their tissues and $5(7 \%)$ cause respiratory ailments.

The highly dangerous species contain alkaloids (9 species: $31 \%$ ), cyanogenic compounds ( 9 species: $31 \%$ ) cardioactive steroids or cardiac glycosides (6 species: $21 \%$ ), toxalbumins (4 species:14\%) or mitotic inhibitors (1 species: $3 \%$ ). Representatives of the categories: anticholinergic poisons, convulsant poisons and sodium channel activators, which are highly dangerous, were not found.

Of the 96 harmful species, the following were encountered most frequently in the educational institutions: Hedera helix L., Pelargonium sp. (Figure 1A) and Schefflera digitata
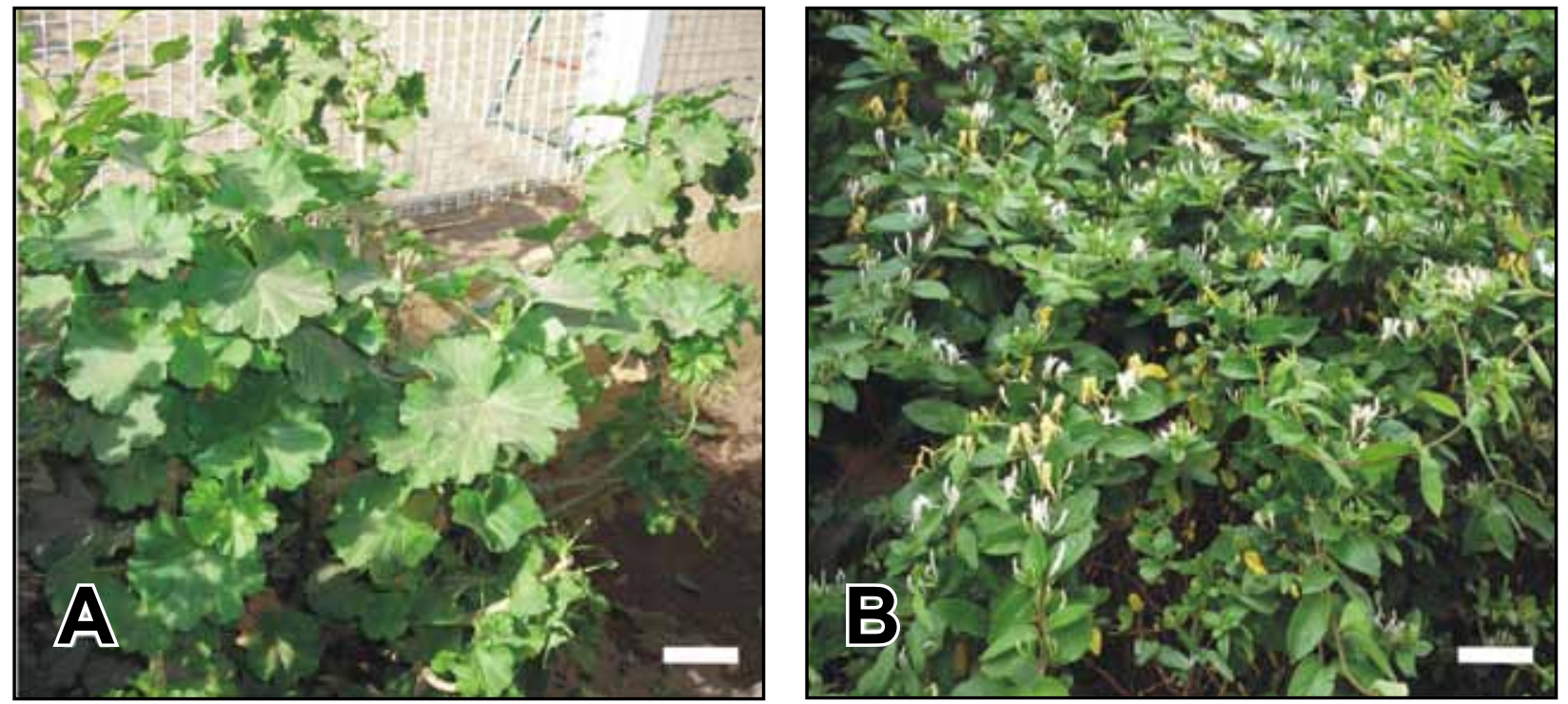

Figure 1. Moderately harmful ornamental species growing in kindergartens of Bahía Blanca City (Argentina). A) Pelargonium sp. (phytodermatitis); B) Lonicera japonica Thunb. (gastrointestinal toxins). Barr: A) $6.53 \mathrm{~cm}$; B) $7.4 \mathrm{~cm}$. 


\section{Pérez Cuadra et al. - Consequences of the Loss of Traditional Knowledge: The risk of injurious and toxic plants growing in kindergartens}
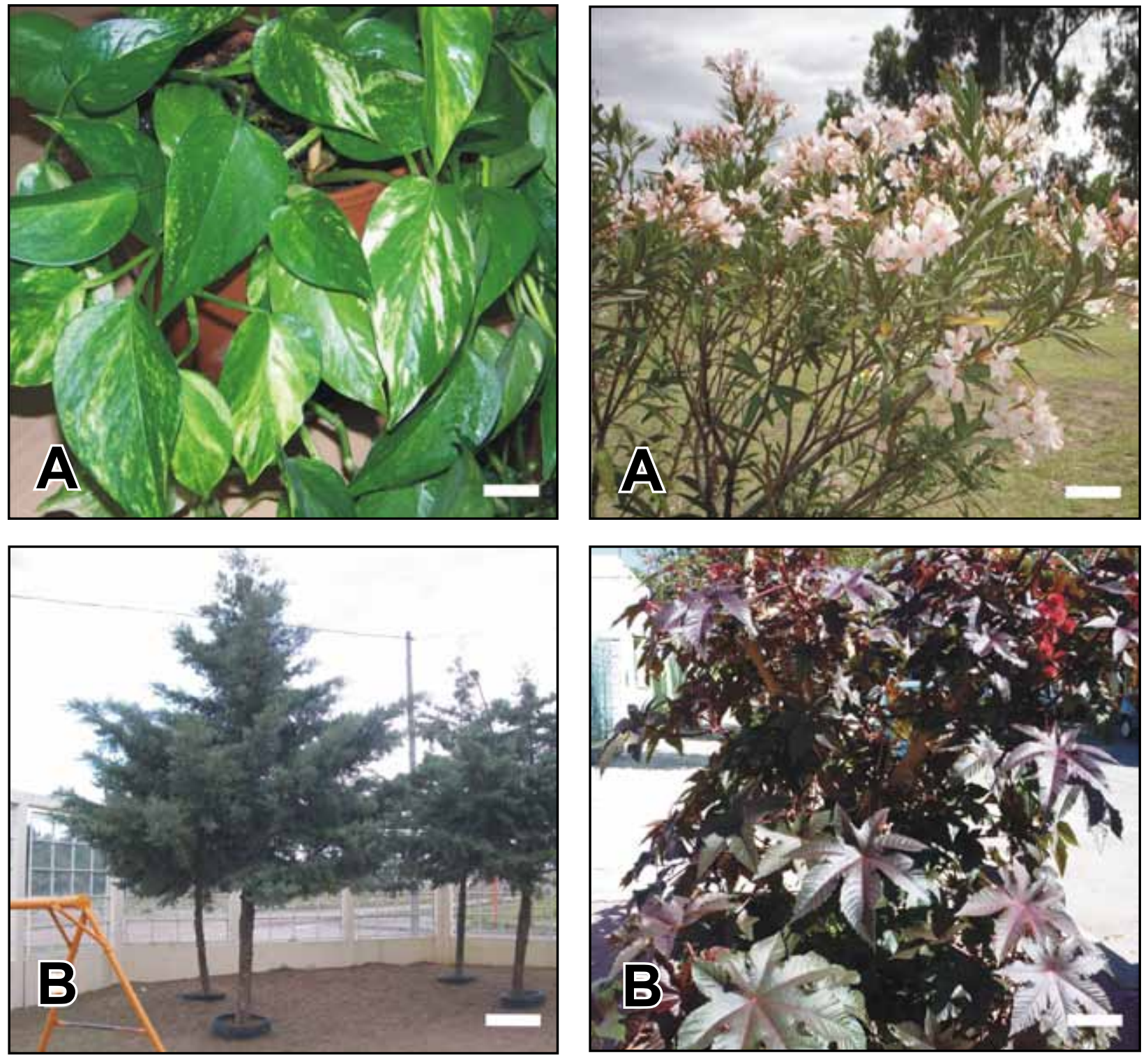

Figure 2. Moderately harmful ornamental species growing in kindergartens of Bahía Blanca City (Argentina). A) Scindapsus aureus (Linden \& André) Engl. \& K. Krause (calcium oxalate crystals); B) Cupressus sp. (respiratory ailments). Barr: A) $3.6 \mathrm{~cm}$; B) $121.7 \mathrm{~cm}$.

The number of ornamental species (harmless, moderately and highly harmful) varies greatly in the kindergartens visited, from 0-45 species. In the same way the proportion of harmless, moderately and highly harmful species was variably: in four institutions there were only harmless species (in those the number of ornamental species ranged between one to five) whereas in 72 kindergartens there were between 20 to $40 \%$ of dangerous ornamental plants and in the other eight, the percentage of these type of plants reach 70 to $80 \%$.

Figure 3. Highly dangerous ornamental species growing in kindergartens of Bahía Blanca City (Argentina). A) Nerium oleander L. (cardioactive steroids or cardiac glycosides); B) Ricinus communis L. (toxoalbumins). Barr: A) $26.6 \mathrm{~cm}$; B) $11 \mathrm{~cm}$.

The species found belonging to the Subclass Polypodiidae (two species) are harmless, while among the plants (301 species) of Subclasses Pinidae and Magnoliidae there are harmless, moderately and highly dangerous plants (Figure 4). Of the species found which belong the Subclass Pinidae (seven species), three are harmless and the others are moderately dangerous (Figure 4), the latter represented by respiratory ailments (three species) and phytodermatitis (one species). The species of Subclass Magnoliidae are the most abundant (294) in all three categories (harmless, moderately and highly dangerous) (Figure 4). Of the 53 species of the Superorder Lilinae, 
Polypodiidae

Pinidae

Asteranae

Buxanae

Caryophyllanae

Lilianae

Magnolianae

Myrothamnanae

Proteanae

Ranunculanae
Rosanae
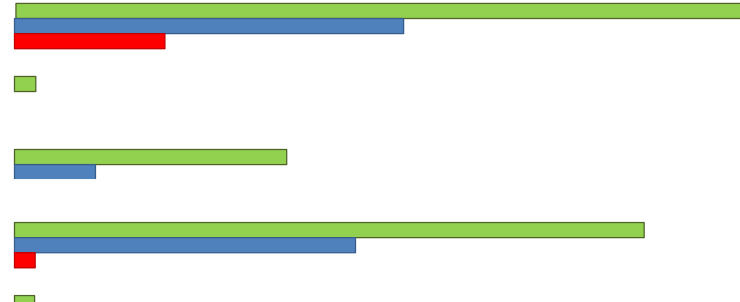

Harmless
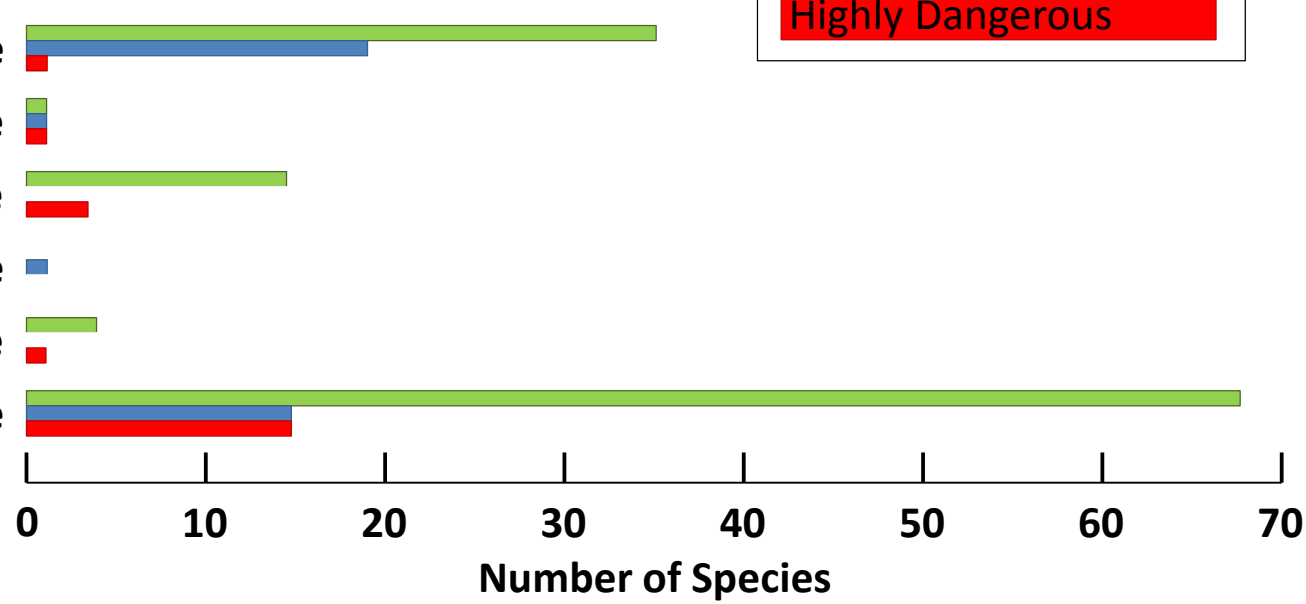

Figure 4. Numbers of harmless, moderately and highly dangerous ornamental species growing in kindergartens of Bahía Blanca City (Argentina).

only one, Clivia miniata Regel, causes serious problems (contains alkaloids) (Table 3), while of the 241 species of the Subclass Magnoliidae, 28 have compounds that are considered highly dangerous represented in the different categories considered (Table 3 ). Of these categories the major represented are cyanogenic compounds (13\%), alkaloids $(11 \%)$ and cardioactive steroids or cardiac glycosides $(8 \%)$. In moderately dangerous species, phytodermatitis $(42 \%)$ and gastrointestinal toxins $(13 \%)$ are those with greater number of representatives (Figure 5).

Ornamental species were found in indoor and outdoor recreational areas, as well as decorating different places in the institutions, e.g., passages, classrooms, kitchens, bathrooms, offices etc. A large number of ornamental plants were located, without any protection (90\%), in places of easy access for children, such as flower beds or flower pots at ground level. In particular, the lower branches of shrubs had not been pruned adequately and the taller children would probably come into contact with them. Outdoor plants were mostly in flower beds, with or without a surrounding protection, whereas indoor plants were all in flower pots of different sizes and materials.

In interviews with the teachers in charge it was evident that most of the institutions (83\%) were not built as kindergartens (Table 4), most of them having previously been private houses, building societies or primary schools, all refurbished and adapted for their present function.
Table 4. Questions and answers from interviews of kindergarten teachers in Bahía Blanca (Argentina).

\begin{tabular}{|l|c|c|c|}
\hline Questions & \multicolumn{3}{|c|}{ Answers } \\
\cline { 2 - 4 } & Yes & No & $\begin{array}{c}\text { Do not } \\
\text { knowl } \\
\text { No } \\
\text { answer }\end{array}$ \\
\hline $\begin{array}{l}\text { Was the property which oper- } \\
\text { ates as a kindergarten designed } \\
\text { and built for this purpose? }\end{array}$ & 15 & 70 & 0 \\
\hline $\begin{array}{l}\text { Were the ornamental species } \\
\text { that are in the kindergarten on } \\
\text { the property at the opening? }\end{array}$ & 60 & 17 & 8 \\
\hline $\begin{array}{l}\text { Was there a landsacaping plan- } \\
\text { ning of the site? }\end{array}$ & 28 & 48 & 9 \\
\hline $\begin{array}{l}\text { Did you have any case of phy- } \\
\text { todermatitis or poisoning caused } \\
\text { by plants? }\end{array}$ & 3 & 82 & 0 \\
\hline $\begin{array}{l}\text { Are you aware of the exis- } \\
\text { tence of dangerous ornamental } \\
\text { plants? }\end{array}$ & 16 & 64 & 0 \\
\hline $\begin{array}{l}\text { Are you interested in learning } \\
\text { more about the subject of orna- } \\
\text { mental dangerous plants? }\end{array}$ & 81 & 2 & 2 \\
\hline $\begin{array}{l}\text { Would you be interested in a sig- } \\
\text { naling of dangerous ornamental } \\
\text { plants? }\end{array}$ & 83 & 2 & 0 \\
\hline
\end{tabular}




\section{Pérez Cuadra et al. - Consequences of the Loss of Traditional Knowledge: The risk of injurious and toxic plants growing in kindergartens}

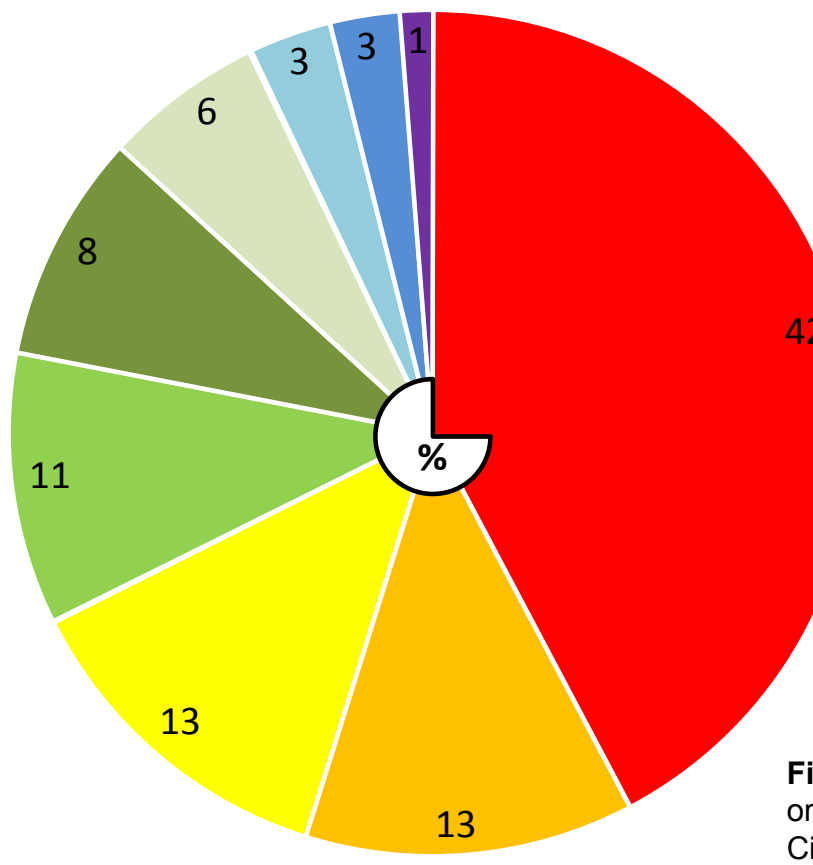

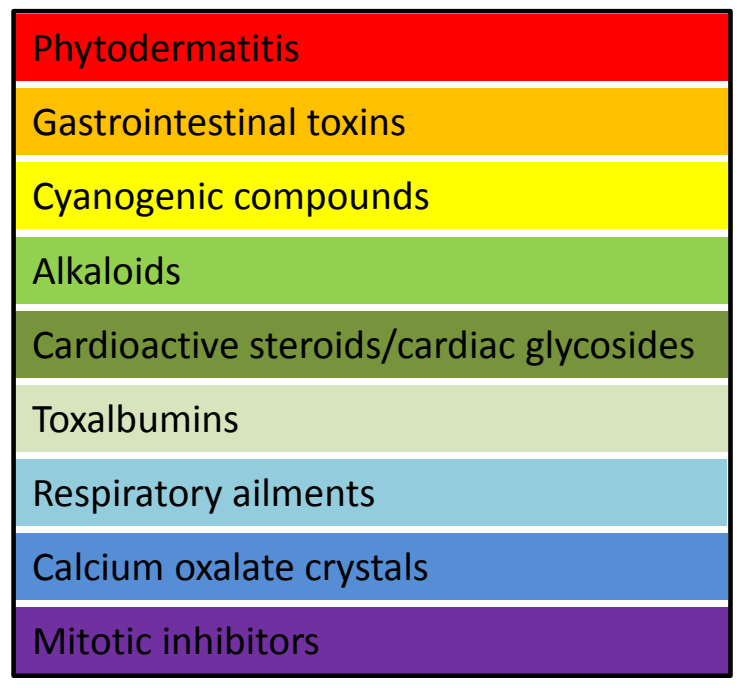

A large percentage $(70 \%)$ of the current plant species were already present when the institutions were inaugurated (Table 4), although others were added later, having been donated or chosen by the personnel. In many kindergartens several plants had been removed to make space for playground equipment.

In most of the educational institutions (57\%) there was no planning for landscaping the grounds (Table 4), but a few had been advised by architects specializing in this area, while, in others, teachers and parents had taken care of the plants. In some institutions there was an initial plan but this had been modified without any control. Most of the plants were chosen to improve the looks of the recreation areas, to provide shade or on account of their rustic nature or adaptability to the climate. In some cases pupils participated in preparing new flower beds by planting seedlings or cuttings.

Medical ailments related to contact with plants were only found in three institutions where the teachers had noticed a child with respiratory problems, one with phytodermatitis due to his allergy to $H$. helix, and one teacher with phytodermatitis. In the first and last cases the causal agent was unknown (Table 4).

However, among the teachers who were interviewed there was a general lack of knowledge about dangerous ornamental plants $(80 \%)$ (Table 4$)$, although some knew of their existence but could not identify or recognise them. Most mentioned the need to learn more about this issue and to label any dangerous species with appropriate signs (Table 4).
Figure 5. Percent of moderately and highly dangerous ornamental species growing in kindergartens of Bahía Blanca City (Argentina) sorted by toxicity categories.

\section{Discussion}

Plants form part of the everyday life of man as they are used for food, clothing, shelter, medicine and, no less importantly, for decorating streets, dwellings and public places, etc. Current technological advances in production processes often make it difficult to recognize the origin of raw materials in any particular product. This has led city dwellers to become more and more out of contact with nature. So we have slowly suffered loss of an understanding of the benefits and dangers of the use of plants and animals for different purposes. This is especially important in the case of plants where there is a thin line between being harmless, medicinal, poisonous or dangerous.

It is commonly thought that plants are harmless, even those with medicinal properties that should be treated with caution as they contain components of pharmacological activity and, although these may be useful for treating certain pathologies, they can also cause adverse effects (Agra et al. 2007, Marinoff et al. 2009). In addition to the medicinal plants there is a large number of poisonous or dangerous plants, possessing chemical compounds which trigger diverse reactions after ingestion or contact with a human being or an animal. These plants are often found in parks and gardens constituting a potential, hidden danger which in many cases is unknown.

This study concentrated on an assessment of dangerous ornamental species in a specific area where children of $0-5$ years of age, the main risk group, spend several hours a day. It should be noted that studies of this kind have not previously been undertaken, at least not specifically 
in Argentina (and generally in South America). This study sets a precedent for the working methodology and it is expected to trigger similar data in different geographical regions, so that better statistics can be obtained of the real percentage of dangerous ornamental species present in the everyday human environment.

The number of harmful species found (95 from 303 species documented) is relatively high (31\%), 30\% (29 species) of these are highly dangerous, whereas the rest normally cause minor injuries. The best represented of the moderately dangerous species are those that cause phytodermatitis because there is a great variety of compounds (oils, latex, etc.) from a host of species that cause this ailment. The most abundant among the highly toxic species are those that contain alkaloids or cyanogenic compounds. It is interesting to mention that only one of this group, $V$. rosea contains mitotic inhibitors which cause severe health problems (Wagstaff 2008). Another very similar species, Vinca major L., is considered harmless as there are no references that verify its toxicity. It is important to emphasize the last statement as the percentage of harmful plants has probably been underestimated since studies on the toxicity of plants are scarce in relation to the estimated number of toxic plants.

The Subclass Magnoliidae includes the largest number of species recorded, this is closely related to the fact that these species are the most commonly cultivated as ornamental, especially those like shrub or herbaceous. To the Subclass Polypodiidae, however, belongs less common species, despite being harmless, which is related to their particular growing requirements. Within the Subclass Pinidae, moderately dangerous species are almost exclusively related to respiratory ailments (one species produces phytodermatitis). In general these types of ailments occur when pollen is released in large amounts in relation with a specific mode of pollination (anemophily). Among the Magnoliidae, particularly within Superorder Lilinae the Araceae family contains all registered species that have calcium oxalate crystals. It should be noted that all species belonging the Superorder Lilinae re moderately dangerous, except $C$. miniata that is the only species that can cause serious damage. Most representatives of the rest of the Superorders of the Subclass Magnoliidae correspond to any of the categories of dangerous ornamental plants found. Among the moderately dangerous, phytodermatitis predominates, while between the highly dangerous, the cyanogenic compounds and the alkaloids are the toxics more frequent. The great variation found between the toxic compounds in these Superorders may relate to the evolutionary radiation of this group of plants, which has led to adapt to different environments and/or biotic interactions. Now that they are cultivated as ornamentals, and in many cases far away from their native habitats, special compounds that allow them to better adapt can cause injury against accidental exposure.
Plants alone do not cause any damage, they have to be in contact with an individual in some way (animal or human) in order to cause an injury. This explains why small children constitute the main risk group of injuries caused by plants, as they come into contact with diverse products including harmful and harmless plants while discovering the environment that surrounds them. In general, it is normal to decorate both homes and kindergartens, inside and outside, with different plant species. During favorable weather children usually spend a large part of the day playing outside in gardens increasing the risk of accidental contact with dangerous plants. In this study it has been shown that, at least in Bahía Blanca, nearly all of the educational institutions have plants that might cause different types of injury and in most cases this risk was unknown, moreover the appropriate treatment in case of poisoning was not known either.

The plants found during this study were, in many cases, already present when the premises were acquired by the educational institution (especially outdoor plants). On the other hand, plants obtained by the institutions were chosen for their beauty, rustic growth, low cost, absence of spines, easy rooting, etc., but their toxicity was never taken into account. This was not due to apathy but to lack of knowledge, as this type of information is not available in nursery gardens, flower shops or supermarkets. So, an additional risk is present, silent and inadvertent, which is not revealed through the inspections required for obtaining authorization to run an educational institution and in most cases this is due to the lack of any appropriately landscape planning of the recreational areas. Harmful species were present at the same percentage in kindergartens that had, and had not, been advised by specialized architects. The intake of toxic plants by children is generally in small quantities, however the fact that the dose of a highly active toxic substance is considerably less for a child than for an adult should be taken into account. This also explains why poisoning is much more common in children (Biondi et al. 2008).

This is particularly important in the accidental consumption of toxic plants. It is difficult to determine how much has been ingested. This underlines the importance of knowing which plants are present in children's playgrounds so that simple and appropriate preventive measures can be taken, e.g., correct taxonomic identification, placing flower pots out of the reach of children, fencing of low areas to prevent contact, or trimming, among others.

Acute poisoning by plants causes symptoms to appear in a short time after exposure to the toxin. Therefore, if the plant is correctly identified it is easier to detect than is the case with chronic poisoning. In the latter it is difficult to attribute the disorder to the ingestion of any plant in particular since the individual is continually in contact with the environment in which there are an infinite number of plants (Díaz Mesa et al. 2009, Marinoff et al. 2009). 


\section{Pérez Cuadra et al. - Consequences of the Loss of Traditional Knowledge: The risk of injurious and toxic plants growing in kindergartens}

Therefore, knowledge of the plants in places where risk groups are found, especially children, is very important for providing the correct diagnosis that leads to rapid and appropriate treatment (Hoffman et al. 2007). As has been mentioned above, three cases of injuries caused by plants were reported from the interviews and only one of them was traced to a particular species $(H$. helix). So, identification of cases is probably underestimated as most people interviewed had not realized that there was any possible connection between an ailment and a plant. In this way in many cases poisoning by plants is not adequately diagnosed and in the general statistics of intoxications it is considered as "poisoning by an unknown cause". In cases that are not reported, or patients do not take into account, or deny that they have ingested a plant, they arrive at a hospital in a critical state and the families know nothing about the reason (Macías Peacok et al. 2009).

\section{Conclusions}

It should be emphasized that poisoning due to plants is accidental (ornamentals) or a result of misuse (medicinal, hallucinogenic or food plants) and they are emergencies that need a multidisciplinary team. An interdisciplinary approach based on different specific academic groups (doctors, pharmacists, biochemists, botanists and biologists) is a key tool for arriving at an accurate diagnosis that ensures the successful resolution of the clinical case (Pérez Cuadra et al. 2010). Poisoning by dangerous ornamental plants occurs throughout the world although until now the issue has not been studied intensively.

Many dangerous ornamental species are cultivated around the world as they tolerate diverse climatic and environmental conditions, e.g., N. oleander (Langford \& Boor 1996), whereas others are natives or endemics of specific regions, e.g., Palicourea longiflora DC. (Coelho et al. 2007). Therefore, it is important to go ahead with the preparation of local guides for the identification of harmful plants.

In Argentina there are no state regulations about the type of species forbidden in landscaping public places or educational institutions. Landscape planning in the courts of kindergartens should be carried out in a way that takes into account the aesthetics and safety of the visitors. Landscapes should be useful for teaching botany in relation to human health and the conservation of nature among other aspects (Biondi et al. 2008). According to current regulations in some regions of Brazil, ornamental plants used in educational institutions should comply with certain criteria, such as rapid growth, adaptation to the climate and soils of the region, and preferably be natives with symbolic characteristics (Biondi et al. 2008). In Argentina, in general, we noticed that when people become alarmed by the presence of dangerous plants (moreover if the plants are cultivated in an educational institution), they instantly try to take them away. This is unnecessary in the majority of cases. On the contrary, it would be interesting to include their recognition in the school curriculum and undertake ways to teach poisoning prevention in different areas of the city (Biondi et al. 2008, Braund \& Reiss 2004). The best way to be safe from this type of poisoning is to know that dangerous plants can be near us. That is why removing them is not the solution. They can appear somewhere else and so this action only contributes to ignorance.

There is very little information on the problems of dangerous ornamental plants written in simple language and accessible to the general public. The role of botanic gardens is fundamental in this respect. A commitment by experts in the design of materials for dissemination is needed that presents the most relevant aspects of the issue. As mentioned by Al-Qura'n (2005), cultivation of well identified toxic plants in botanic gardens as a didactic resource is a most important activity. Botanic gardens could integrate work on conservation, research and education as it is the appropriate place and they have the infrastructure and organization for such purposes. Medicinal plants have already been included in actions of this type as their value is known and there is information about their use and preservation (Hawkins 2008). All of these actions should be accompanied by special preventative policies for each region that strengthen the commitment of all stakeholders in the issue.

Researchers emphasize that the most urgent and effective measures to be taken are prevention and education (dissemination to the community in general and to doctors) in order to reduce the number of accidents involving plants (Botha \& Penrith 2008, Hoffman et al. 2007, Rocha Silva \& Takemura 2006). Certainly these aspects are very important and they will enable major advances in this issue, but, due to the lack of knowledge on the current situation locally and regionally, it is extremely important and necessary to work in greater depth, as proposed here, in order to diagnose the scenario and then plan future actions that would integrate interdisciplinarity, scientific research and community outreach.

The quantification of dangerous plants made in this work in places frequented by high-risk groups (young children) is the first step in Argentina which will allow an advance in the diagnosis of the current situation. The rise of the number of studies, as is proposed here, will allow development of specific preventive policies at the local level, because the ornamental species that are used are often related to local customs. Then, prevention policies at both regional and national levels should be laid out, to build a consensus regarding the regulations about the planning of landscaping for certain areas (specially those engaged with high-risk population like young children). The commitment of the educators is needed, to integrate the knowledge and to promote prevention. Finally, global strategies 
of dissemination of the importance of knowing the plant world, that are part of the everyday life of every person in the world, should be generated.

In this way, during the development of this study, the Jefatura Distrital de Educación of Bahía Blanca City (dependent of the Ministerio de Educación of Buenos Aires Province) in charge of the kindergartens of the Partido de Bahía Blanca, promoted the realization of a workshop on dangerous plants of obligatory character for Directors of each Institution. All Directors received a photographic atlas (Cambi \& Pérez Cuadra 2010) to facilitate the recognition of the plants in their Institutions. Similarly, a Deputy of Buenos Aires Province presented a project requesting floristic inventories in primary schools. This little light that was ignited during the development of the project is a clear sign that we are beginning the way.

\section{Literature Cited}

Agra, M.F., P. França de Freitas \& J.M. Barbosa Filho. 2007. Sinopsis of the plants known as medicinal and poisonous in Northeast of Brazil. Revista Brasileira de Farmacognosia 17:114-140.

Al-Quara'n, S. 2005. Ethnobotanical survey of folk toxic plants in southern part of Jordan. Toxicon 46:119-129.

Álvarez Arias, B.T. 2000. Ichthyotoxic plants used in Spain. Journal of Ethnopharmacology 73:505-512.

Armitage, A.M. 2004. Armitage's Garden Annuals. Timber Press, Portland, Oregon.

Armitage, A.M. 2011. Armitage's Manual of Annuals, Biennials and Half Hardy Perennials. Timber Press, Portland, Oregon.

Austin, C. 2005. Irises. Timber Press, Portland, Oregon.

Biondi, D., L. Leal \& M. Schaffer. 2008. Aspectos importantes das plantas ornamentais em escolas públicas estaduais da cidade de Curitiba, PR. Revista Brasileira de Ciências Agrárias 3:267-275.

Bofill, F.X., J. Bofia, G. Such, E. Piqué \& R. Guitart. 2007. Dos casos de intoxicación por contaminación de maíz con Datura stramonium en ganado vacuno. Revista de Toxicología 24:56-58.

Board, P.G., M. Coggan, G. Chelvanayagam, S. Easteal, L.S. Jermiin, G.K. Schulte, D.E. Danley, L.R. Hoth, M.C. Griffor, A.V. Kamath, M.H. Rosner, B.A. Chrunyk, D.E. Perregaux, C.A. Gabel, K.F. Geoghegan \& J. Pandit. 2000. Identification, characterization, and crystal structure of the omega class glutathione transferases. The Journal of Biological Chemistry 275:24798-24806.
Botha, C.J. \& M.L. Penrith. 2008. Poisonous plants of veterinary and human importance in southern Africa. Journal of Ethnopharmacology 119:549-558.

Braund, M. \& M. Reiss. 2004. Learning Science Outside the Classroom. Routledge Falmer, London.

Bryan, J.E. 2002. Bulbs. Timber Press, Portland, Oregon.

Cambi, V. \& V. Pérez Cuadra. 2010. Conozcamos las Plantas: Galería fotográfica de ornamentales presentes en los Jardines de Infantes del Partido de Bahía Blanca (Arg.). Autoras editoras, Bahía Blanca, Argentina.

Coelho, E.G., A.C.F. Amaral, J.L.P. Ferreira, A.G. dos Santos, M.L.B. Pinheiro \& J. Rocha de A. Silva. 2007. Calcium oxalate crystals and methyl salicylate as toxic principles of the fresh leaves from Palicourea longiflora, an endemic species in the Amazon state. Toxicon 49:407-409.

Córdoba, A.P., B. Soto Vallejo, C.A.G. Polo, M.G. Isaza \& A.J.H. Gallego. 2003. Plantas tóxicas caseras en la ciudad de Manizales. Biosalud 2:15-29.

Crosby, D.G. 2004. The Poisoned Weed: Plants toxic to the skin. Oxford University Press, New York, New York.

Díaz Mesa, A., E. Pereira Valdés, A. Enseñat Álvarez \& C.A. Rodríguez Armada. 2009. Guía de práctica clínica para las intoxicaciones exógenas. Revista Electrónica de las Ciencias Médicas de Cienfuegos 7:96-100.

Dimitri, M.J. 1988. Encyclopedia Argentina de Agricultura y Jardinería. ACME, Buenos Aires.

Dirr, M.A. 2002. Dirr's Trees and Shrubs for Warm Climates. Timber Press, Portland, Oregon.

Freire S.E., A.M. Arambarri, N.D. Bayón, G. Sancho, E. Urtubey, C. Monti, M.C. Novoa \& M.N. Colares. 2005. Epidermal characteristics of toxic plants for cattle from the Salado river basin (Buenos Aires, Argentina). Boletín de la Sociedad Argenina de Botánica 40(3-4):241-281.

Gallo, G.G. 1987. Plantas tóxicas para el ganado en el cono sur de América. Hemisferio Sur, Buenos Aires.

Hawkins, B. 2008. Plants for Life: Medicinal plant conservation and botanic gardens. Botanic Gardens Conservation International, Richmond, United Kingdom.

Hoffman, R.S., L.S. Nelson, M.A. Howland, N.A. Lewin, N.E. Flomenbaum \& L.R. Goldfrank. 2007. Goldfrank's Manual of Toxicologic Emergencies. McGraw-Hill, New York, New York. 


\section{Pérez Cuadra et al. - Consequences of the Loss of Traditional Knowledge: The risk of injurious and toxic plants growing in kindergartens}

Hurrell, J., D. Bazzano \& G. Delucchi. 2006. Biota Rioplatense XI: Dicotiledóneas herbáceas 1. LOLA, Buenos Aires.

Hurrell, J., D. Bazzano \& G. Delucchi. 2007. Biota Rioplatense XII: Dicotiledóneas herbáceas 2. LOLA, Buenos Aires.

INDEC. 2001. Instituto Nacional de Estadísticas y Censos. República Argentina. www.indec.mecon.ar/webcenso/index.asp (verified 5 March 2011).

Innes, C. \& C. Glass. 1997. The Illustrated Encyclopedia of Cacti. Knickerbocker Press, New York, New York.

Iramain, M.S., M.A. Herrero, S.M. Volpe \& S.E. Toro. 2008. Plantas Ornamentales Tóxicas. BMPress, Ciudad Autónoma de Buenos Aires.

Langford, S.D. \& P.J. Boor. 1996 Oleander toxicity: An examination of human and animal toxic exposures. Toxicology 109:1-13.

Le Coz, C.J., G. Ducombs \& E. Paulsen. 2011. Plants and plant products. Pp. 873-925 in Contact Dermatitis. Edited by J.D. Johansen, P.J. Frosch \& J.P. Lepoittevin. Springer, Berlin, Germany.

Macías Peacok, B., M.F. Suárez Crespo, C.A. Berenguer Rivas \& L. Pérez Jackson. 2009. Intoxicaciones por plantas tóxicas atendidas desde un servicio de información toxicológica. Revista Cubana de Plantas Medicinales 14:1-8.

Marinoff, M.A., J.L. Martínez \& M.A. Urbina. 2009. Precauciones en el empleo de plantas medicinales. Boletín Latinoamericano y del Caribe de Plantas Medicinales y Aromáticas 8:184-187.

Morren, M.A. \& A. Goossens. 2011. Contact allergy in children. Pp. 937-961 in Contact Dermatitis. Edited by J.D. Johansen, P.J. Frosch \& J.P. Lepoittevin. Springer, Berlin, Germany.

Nájera, M.T. 1993. Aportes al conocimiento de las plantas tóxicas (I parte). Rojasiana 1:43-48.

Nelson, L.S., R.D. Shih \& M.J. Balick. 2007. Handbook of Poisonous and Injurious Plants. Springer, New York, New York.

Neuwinger, H.D. 2004. Plants used for poison fishing in tropical Africa. Toxicon 44:417-430.

Noonan, S.C. \& G.P. Savage. 1999. Oxalate content of foods and its effect on humans. Asia Asia Pacific Journal of Clinical Nutrition 8:64-74.
Llamas, K.A. 2003. Tropical Flowering Plants. Timber Press, Portland, Oregon.

Olsnes, S. 2004. The history of ricin, abrin and related toxins. Toxicon 44:361-370.

Pérez Cuadra, V., F. Turano, V. Cambi \& M.A. Rueda. 2010. Plantas medicinales y ornamentales tóxicas. Revista de la Asociación Médica de Bahía Blanca 20:67-70.

Pinillos, M.A., J. Gómez, J. Elizalde \& A. Dueñas. 2003. Intoxicación por alimentos, plantas y setas. Anales del Sistema Sanitario de Navarra 26:243-263.

Rates, S.M.K. 2001. Plants as source of drugs. Toxicon 39:603-613.

Robles C.A., C. Saber \& M. Jefrey. 2000. Intoxicación por Astragalus pehuenches (locoismo) en ovinos Merino de la Patagonia Argentina. Revista de Medicina Veterinaria 81(5): 380-384

Rocha Silva, I.G. \& O.S. Takemura. 2006. Aspectos de intoxicações por Dieffenbachia sp. (Comigo-ninguémpode) - Araceae. Journal of Medical and Biological Sciences 5:151-159.

Sajeva, M. \& M. Costanzo. 2001. Succulents, the Illustrated Dictionary. Timber Press, Portland, Oregon.

Schmid, W.G. 2002. An Encyclopedia of Shade Perennials. Timber Press, Portland, Oregon.

Siener, R., R. Hönow, A. Seidler, S. Voss \& A. Hesse 2006. Oxalate contents of species of the Polygonaceae, Amaranthaceae and Chenopiaceae families. Food Chemistry 98:220-224.

Szpunar, J. 2005. Advances in analytical methodology for bioinorganic speciation analysis: metallomics, metalloproteomics and heteroatom-tagged proteomics and metabolomics. Analyst 130:442-465.

Stegelmeier, B.L., J.A. Edgar, D.R. Gardner, R.A. Coulombe \& R.J. Molyneux. 1999. Pyrrolizidene alkaloid plants, metabolism and toxicity. Journal of Natural Toxins 8:95-116.

Tropicos.org. 2011. Missouri Botanical Garden. www.tropicos.org (verified 21 September 2011).

Turner, N.J. \& A.F. Szcawinski. 1991. Common Poisonous Plants and Mushrooms of North America. Timber Press, Portland, Oregon.

Wagstaff, D.J. 2008. International Poisonous Plants Checklist. An evidence-based reference. CRC Press, Boca Ratón, Florida. 
Zachos, E. 2008. Tempting Tropicals. Timber Press, Portland, Oregon.
Zeinsteger, P.A \& A.A. Gurni. 2004. Plantas tóxicas que afectan el aparato digestivo de caninos y felinos. Revista veterinaria $15: 35-44$. 